\title{
Transcriptional control of monocyte gene expression in post-traumatic stress disorder
}

\author{
Aoife O’Donovan ${ }^{\mathrm{a}, \mathrm{b}, \mathrm{c}, *}$, Bing Sun $^{\mathrm{a}, \mathrm{b}, \mathrm{d}}$, Steve Cole ${ }^{\mathrm{e}}$, Hans Rempel $^{\mathrm{a}, \mathrm{b}}$, Maryann Lenoci $^{\mathrm{a}, \mathrm{b}, \mathrm{c}}$, \\ Lynn Pulliam ${ }^{\mathrm{a}, \mathrm{b}, \mathrm{d}}$ and Thomas Neylan ${ }^{\mathrm{a}, \mathrm{b}, \mathrm{c}}$ \\ ${ }^{a}$ Veteran's Affairs Medical Center, San Francisco, CA, USA \\ ${ }^{\mathrm{b}}$ Northern California Institute for Research and Education, San Francisco, CA, USA \\ ${ }^{\mathrm{c}}$ Department of Psychiatry, University of California, San Francisco, CA, USA \\ ${ }^{\mathrm{d}}$ Department of Laboratory Medicine, University of California, San Francisco, CA, USA \\ ${ }^{\mathrm{e}}$ Department of Medicine, University of California, Los Angeles, CA, USA
}

\begin{abstract}
Post-traumatic stress disorder (PTSD) confers an increased risk for disorders with an inflammatory etiology. PTSDrelated dysregulation of the sympathetic nervous system (SNS) and hypothalamic-pituitary adrenal (HPA) axis and associated alterations in inflammatory activity may contribute to this increased risk. However, little is known about convergent SNS, HPA and inflammatory signaling at the level of the immune cell transcriptome in PTSD. To explore such signaling, we examined the prevalence of specific transcription factor binding motifs in the promoter regions of differentially expressed genes in monocytes from individuals with PTSD and matched controls. Participants included 49 men (24 PTSD+ and 25 trauma-exposed controls) and 18 women (10 PTSD+ and 8 controls). Men with PTSD showed up-regulation of target genes for the NF- $\kappa \mathrm{B} / \mathrm{Rel}$ family of transcription factors, which convey inflammatory signals, up-regulation of target genes for CREB/ATF transcription factors, which convey adrenergic signals from the SNS, and down-regulation of target genes for the glucocorticoid receptor, which conveys glucocorticoid signals from the HPA axis. Women with PTSD also showed significant up-regulation of target genes for NF- $\kappa$ B and non-significant down-regulation of target genes for GR, but significant down-regulation of target genes for CREB/ATF. Altered transcriptional control of monocyte gene expression could contribute to exaggerated inflammatory activity in PTSD.
\end{abstract}

\section{Introduction}

Post-traumatic stress disorder (PTSD) is associated with substantially increased risk for chronic diseases such as neurodegenerative disorders, cardiovascular disease, diabetes, asthma, and arthritis $[8,10,49$, 52,59]. Importantly, this PTSD-related increased risk for disease does not appear to be accounted for by potential confounds and mediators such as family history, smoking, obesity, alcohol dependence, or depression [5]. PTSD also confers more than two-fold higher risk for early mortality [5,7], and the increased risk for early mortality associated with PTSD is comparable to that of more traditional disease indicators such as

* Corresponding author: Aoife O'Donovan, Department of Psychiatry, University of California, San Francisco, San Francisco Veteran's Affairs Medical Center, San Francisco CA 94121, USA. Tel.: +1 4152214810 (Extn: 4959); E-mail: aoife.odonovan@ucsf.edu. elevated white blood cell count and erythrocyte sedimentation rate [6]. Although the biological underpinnings of PTSD are poorly understood, dysregulation of immune system processes could partially mediate PTSD-related increased risk for disease.

Elevated inflammatory activity is increasingly recognized as a potential pathway to PTSD-related increased risk for physical disease. Diseases that are more common in individuals with PTSD share an inflammatory etiology [4,59], and preliminary evidence indicates that PTSD is associated with increased levels of pro-inflammatory proteins including cytokines and C-reactive protein $[22,53,57]$. Moreover, a large body of research indicates that PTSD is associated with dysregulation of two systems that are known to influence inflammatory activity, specifically the sympathetic nervous system (SNS) and the hypothalamic-pituitary adrenal (HPA) axis [27,50,51]. However, the precise nature of this dysregulation remains incompletely un- 
derstood with some studies reporting up-regulation, some down-regulation and some equivalent levels of SNS and HPA axis activity in individuals with PTSD compared with controls $[23,40,44,56,63]$.

Most, but not all, studies have documented increased SNS activity in PTSD, as indexed by increased sympathetic and decreased parasympathetic tone and elevated levels of peripheral catecholamines $[40,56,65]$. A more complex story has emerged regarding HPA axis activity. PTSD has generally been associated with elevated levels of corticotrophin-releasing hormone (CRH), indicating increased activation of the HPA axis [25,38], but with lower or equivalent levels of the glucocorticoid hormone cortisol, indicating decreased activation of the HPA axis [37,64]. A widely validated explanation for these conflicting findings is found in studies reporting enhanced negative feedback inhibition by cortisol in individuals with PTSD $[38,43,62]$. This enhanced negative feedback inhibition is thought to be mediated through higher responsiveness or sensitivity of glucocorticoid receptors (GR) [60], and is consistent with the observed pattern of elevated $\mathrm{CRH}$ in combination with lower cortisol. This theoretical model also underlines the importance of examining signaling pathways involved in regulating the activity of cells, including immune cells, in PTSD.

The activity of immune cells is mediated by transcription factors that bind to DNA and regulate the expression of genes. The nuclear factor- $\kappa \mathrm{B}(\mathrm{NF}-\kappa \mathrm{B}) / \mathrm{Rel}$ family of transcription factors plays a crucial role in regulating the expression of genes involved in the inflammatory response by transmitting receptor signals to the nucleus $[1,21,41]$. The first class of NF-kB/Rel transcription factors includes NF- $\kappa \mathrm{B} 1$ (p50) and NF$\kappa \mathrm{B} 2$ (p52), which can only regulate transcription when they form dimers with members of the second class of NF- $\kappa \mathrm{B} / \mathrm{Rel}$ transcription factors. The second class includes RelA (p65), RelB and c-Rel, which can directly activate transcription of many genes, including those involved in inflammatory activity. NF- $\kappa \mathrm{B} / \mathrm{Rel}$ transcription factors are activated by viruses, gramnegative bacteria and inflammatory cytokines [58], and also in response to psychological stress [2]. Inflammatory activity is additionally subject to regulation by catecholamines and glucocorticoids, which bind to adrenergic receptors and GR on immune cells respectively $[15,16]$. Such binding is followed by signaling to the transcriptome by associated transcription factors including cyclic adenosine monophosphate response element binding protein (CREB) and activating transcription factor (ATF), which convey adrenergic (and other) signals, and GR, which conveys glucocorticoid signals. Observed elevations in inflammatory activity in PTSD may thus be due to altered signaling by NF- $\kappa \mathrm{B} / \mathrm{Rel}$, CREB/ATF and GR in immune cells. However, little is known about alterations in these transcriptional control pathways in PTSD.

In the present study, we analyzed monocyte gene expression data obtained from patients with PTSD and control participants using the Transcription Element Listeining System (TELiS) [13]. Monocytes were chosen for these gene expression analyses because they are influenced by psychosocial factors [19,31], bear receptors for catecholamines and glucocorticoids [20], and respond to immune challenges by producing inflammatory proteins [33]. TELiS permits examination of the promoter regions of differentially expressed genes for enrichment with transcription factor binding motifs (TFBMs) or response elements for specific transcription factors (see Fig. 1 for overview). We specifically examined if differentially expressed genes in monocytes from patients with PTSD were significantly enriched with response elements for NF- $\kappa \mathrm{B}, \mathrm{CREB} / \mathrm{ATF}$ and GR in separate male and female samples.

\section{Methods}

\subsection{Sample}

Medically healthy participants were recruited from the Bay Area community via advertisements in public settings and from the San Francisco Veterans Affairs Medical Center. Participants included 49 men $(n=24$ PTSD+; $n=25$ PTSD- Control) and 18 women ( $n=$ 10 PTSD+; $n=8$ PTSD- Control). Diagnoses were made by trained interviewers using structured clinical interviews. Controls were age matched and in the case of the male sample, were also matched on trauma exposure. Baseline laboratory tests included a complete blood count, serum chemistry panel, liver and thyroid function tests, serology for Hepatitis B \& C, urine toxicology screen, and urine pregnancy test (if appropriate). Tests indicated that all participants were medication free, medically healthy and without current infection. Participants had normal white blood cell counts, no elevations in body temperature, negative serology for HIV and hepatitis, and no current substance use disorders. All participants provided written informed consent and the study protocol was approved by the Committee on Human Research at the University of California, San Francisco and at the San Francisco Veteran's Affairs Medical Center. 


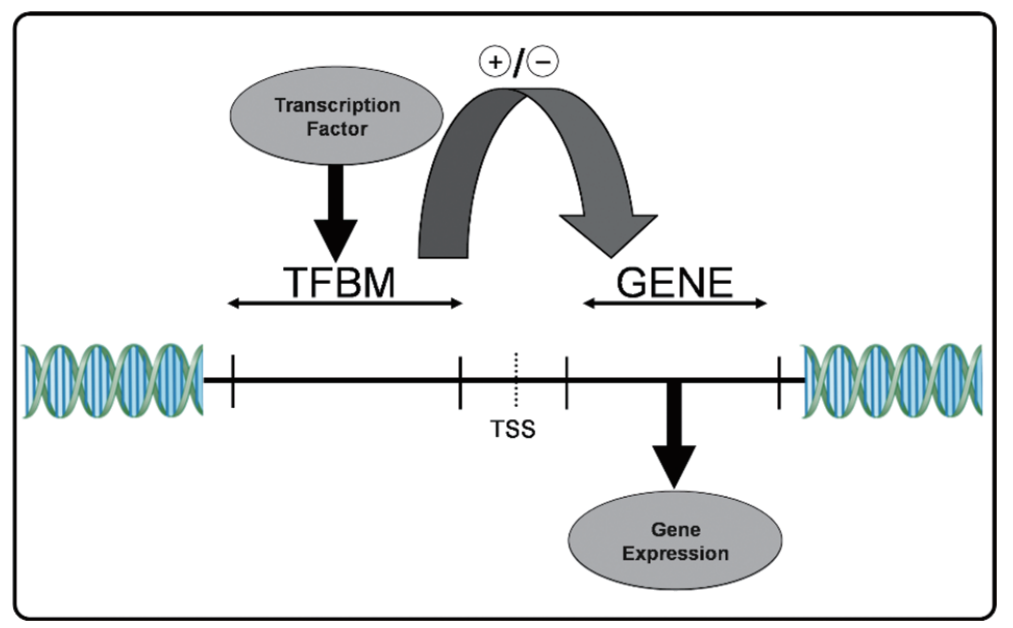

Fig. 1. Promoter regions of genes include DNA sequences called response elements or transcription factor binding motifs (TFBMs) that are specific to particular transcription factors. Binding of transcription factors to these TFBMs can inhibit or promote expression of a gene, ultimately determining the activity of the cell. The Transcription Element Listening System (TELiS) [13] uses bioinformatic techniques to examine the promoter regions of differentially expressed genes for enrichment with TFBMs for specific transcription factors of interest.

\subsection{Measures}

Self-report questionnaires were used to determine demographic details. A structured clinical interview was used to assess lifetime and current anxiety and mood disorders, PTSD and history of stressful life events. Interview assessments included the Structured Clinical Interview for DSM-IV, Patient edition (SCIDP) to assess mood disorders, substance use disorders, and anxiety disorders [17], and the Clinician Administered PTSD Scale (CAPS) to assess trauma exposure as well as presence and severity of PTSD [3]. Trained interviewers diagnosed all patients and weekly case consensus meetings were used to confirm diagnoses.

\subsection{Monocyte gene expression analysis}

For monocyte gene expression analysis, sixty milliliters of blood was drawn into cell preparation tubes (Becton Dickinson) and centrifuged to enrich for monocytes by magnetic separation using MACS CD14 microbeads (Miltenyi Biotech, Auburn, CA). Total RNA was isolated from CD14+ monocytes using Qiagen RNeasy Micro Kit (Qiagen, Valencia, CA) following the manufacturer's protocol and stored at $-70 \mathrm{C}$. The purity and concentration of the RNA was determined with spectrophotometer, and the integrity of the RNA was determined with an RNA picochip on an Agilent Bioanalyzer 2100 (Agilent Technologies Inc, Palo Alto, CA). CodeLink Human Whole Genome BioArrays were used to identify genes differentially ex- pressed in CD14+ monocytes between participants with PTSD and controls [48]. The BioArrays have a total of 54,841 probes of which $83 \%$ of the represented genes are clearly annotated (based on unique UniGene IDs), $39 \%$ well annotated (categorized to a gene ontology) and $53 \%$ have been mapped to specific chromosome locations. One half microgram of total RNA isolated from CD14+ monocytes was amplified and labeled with biotin-11-UTP (Perkin Elmer, Boston, MA) using the CodeLink iExpress iAmplify Kit (AMI). Ten micrograms of fragmented cRNA were hybridized to microarrays using the procedures suggested by the manufacturer. The arrays were scanned on an Axon GenePix 4000B scanner (Molecular Devices, Sunnyvale, CA). Image analysis and data extraction were performed by CodeLink Expression Software Kit v4.1 (AMI). Following standardization and normalization of the raw data using loess normalization in R/BioConductor, differentially expressed genes from the PTSD and control subjects were evaluated for fold change.

\subsection{Transcriptional control pathways analysis}

The GeneSpring GX 7.3 software package (Agilent, Santa Clara, CA) together with the Bioconductor [18] suite of $\mathrm{R}$ packages [54] were used for microarray data analysis [39]. To examine if differential monocyte gene expression in participants with PTSD might stem from differences in upstream inflammatory, adrenergic or glucocorticoid transcriptional activity, we performed bioinformatics analysis of promot- 
er DNA sequences using TELIS software (http://www. telis.ucla.edu/) [13]. Differentially expressed genes were identified as those that were significantly different between participants with PTSD and controls on Student's t-test $(p<0.05)$ with at least $30 \%$ difference in levels of expression. Using the transcriptional shift analysis variant of TELiS, we examined differential expression of genes bearing response elements for specific response elements included in the TRANSFAC database [26]. Analyses for the NF$\kappa \mathrm{B} / \mathrm{Rel}$ family of transcription factors were focused on response elements for NF- $\kappa \mathrm{B} /$ Rel transcription factors that can independently convey pro-inflammatory signals, specifically V\$NFKAPPAB65_01 for RelA (p65) and V\$CREL_01 for c-Rel. Analyses for the CREB/ATF family of transcription factors were focused on V\$CREB_01 for CREB-1 and V\$ATF_01 for ATF-1, which are elements responsive to adrenergic signals from catecholamines [45]. Analyses for GR were focused on V\$GR_Q6, a response element for GR- $\alpha$ and GR- $\beta$. In exploratory analyses, we examined differential expression of genes bearing response elements for other NF- $\kappa$ B/Rel and CREB/ATF transcription factors. Analyses used aggregate indices that had been pooled across 9 different technical specifications involving variations of promoter length and TFBM match stringency. Differential representation of TFBMs was tested using an independent sample t test with Welch's correction for heteroscedasticity.

\section{Results}

\subsection{Male sample characteristics}

The sample of men with PTSD (PTSD+) and control participants were comparable with regard to age (PTSD+ $M$ Age $=30, S D=6$; Control $M$ Age $=$ $30, S D=6 ; p=0.85$ ) and marital status (PTSD+ $50 \%$ single, $25 \%$ married and $25 \%$ divorced; Control $56 \%$ single, $32 \%$ married and $12 \%$ divorced; $p=0.32$ ). Overall, the male sample was ethnically heterogeneous, including African American $(n=5)$, Asian $(n=4)$, Caucasian $(n=26)$, Hispanic $(n=8)$, Middle Eastern $(n=2)$, Pacific Islander $(n=2)$, and mixed race $(n=2)$ participants in both PTSD+ and control groups. There were no significant group differences in ethnicity $(p=0.68)$. More detailed demographic information can be found in [39]. Trauma exposure and PTSDrelated information is presented in Table 1 where it can be seen that men with PTSD and control participants had been exposed to similar categories of trauma. Further, it can be seen that men with PTSD had significantly higher current scores on the CAPS than control participants. All control participants were negative for a lifetime diagnosis of PTSD.

\section{Male transcriptional control pathways}

To examine if differentially expressed genes were significantly enriched with specific response elements of interest, we used TELiS to search for NF- $\kappa \mathrm{B}$, CREB/ATF and glucocorticoid binding motifs in the promoter regions of differentially expressed genes. Using a cutoff point of 1.3 fold difference in gene expression levels, 13 up-regulated and 53 down-regulated genes were included in analyses comparing male participants with PTSD with age- and sex-matched controls (Supplementary Table 1 and cf. [39]).

Among men with PTSD, there was significant upregulation of genes with response elements for the NF$\kappa \mathrm{B} /$ Rel transcription factors, RelA (1.55-fold difference in V\$NFKAPPAB65_01, $\mathrm{SE}=0.22, p=0.01)$ and c-Rel (1.42-fold difference in V\$CREL_01, SE = $0.13, p=0.004)$. Second, there was significant upregulation of genes with response elements for CREB1 (1.50-fold difference in V\$CREB_01, $\mathrm{SE}=0.18, p=$ 0.006) and ATF-1 (1.79-fold difference in V\$ATF_01, $\mathrm{SE}=0.29, p=0.005)$. Finally, there was significant down-regulation of genes with response elements for GR- $\alpha$ and GR- $\beta$ (0.73-fold difference in V\$GR_Q6, SE $=0.06, p=0.01$ ). Figure 2a illustrates these findings.

In exploratory analyses, we also found significant up-regulation of genes with response elements for $\mathrm{NF}-\kappa \mathrm{B}, \mathrm{NF}-\kappa \mathrm{B} 1, \mathrm{NF}-\kappa \mathrm{B} 2$ (1.35-fold difference in $\left.\mathrm{V} \$ N F K B \_C, \mathrm{SE}=0.15, p=0.04\right)$ and up-regulation of genes with response elements for three additional CREB/ATF transcription factors (2.46-fold difference in V\$CREBP1CJUN_01, SE $=0.56, p=0.002 ; 1.84$ fold difference in V\$CREB_Q4, SE $=0.40, p=0.01$; 1.38 -fold difference in V\$CREB_Q2, $\mathrm{SE}=0.17, p=$ $0.02)$.

\section{Female sample characteristics}

Female PTSD+ participants were age-matched with control participants. The final sample of female PTSD+ and control participants were comparable with regard to age (PTSD+ $M$ Age $=30, S D=7$; Control $M$ Age $=27, S D=6 ; p=0.33$ ) and non-significantly 
Table 1

Clinical characteristics of male and female samples

\begin{tabular}{|c|c|c|c|c|c|c|}
\hline & \multicolumn{3}{|c|}{ Male } & \multicolumn{3}{|c|}{ Female } \\
\hline & $\mathrm{PTSD}+(N=24)$ & Control $(N=25)$ & $\mathrm{p}$ & $\mathrm{PTSD}+(N=10)$ & Control $(N=8)$ & $\mathrm{p}$ \\
\hline Trauma Exposure & 0 & & & & & \\
\hline Combat & $16(67)$ & $12(48)$ & & 0 & 0 & \\
\hline Physical abuse/assault & $6(25)$ & $5(20)$ & & $6(60)$ & 0 & \\
\hline Sexual abuse/assault & $1(4)$ & 0 & & $4(40)$ & $1(12)$ & \\
\hline Accident & 0 & $2(8)$ & & 0 & $1(12)$ & \\
\hline Robbery & $1(4)$ & $2(8)$ & & 0 & 0 & \\
\hline Other & 0 & $4(16)$ & 0.25 & 0 & 0 & $0.04^{*}$ \\
\hline Current CAPS M (SD) & $57(15)$ & $4(6)$ & & $64(18)$ & $2(3)$ & \\
\hline Intrusion & $15(5)$ & $1(3)$ & & $16(6)$ & 0 & \\
\hline Avoidance & $19(7)$ & $1(2)$ & & $26(11)$ & 0 & \\
\hline Hyperarousal & $22(7)$ & $2(3)$ & $0.000^{*}$ & $21(4)$ & $2(3)$ & $0.001^{*}$ \\
\hline Lifetime CAPS M (SD) & $\mathrm{n} / \mathrm{a}$ & $8(10)$ & & $\mathrm{n} / \mathrm{a}$ & $13(18)$ & \\
\hline Intrusion & & $4(6)$ & & & $9(13)$ & \\
\hline Avoidance & & $1(2)$ & & & 0 & \\
\hline Hyperarousal & & $3(1)$ & $\mathrm{n} / \mathrm{a}$ & & $4(4)$ & $\mathrm{n} / \mathrm{a}$ \\
\hline
\end{tabular}

Notes. n/a indicates not applicable and $*$ denotes statistical significance at $p<0.05 . P$ values are based on Student's t-tests for continuous data and on Mann Whitney U tests for categorical data. For trauma exposure, numbers refer to $n(\%)$. The mean current and lifetime CAPS scores for females are based on only two participants who had experienced a traumatic event fulfilling Criterion A of the CAPS measure.

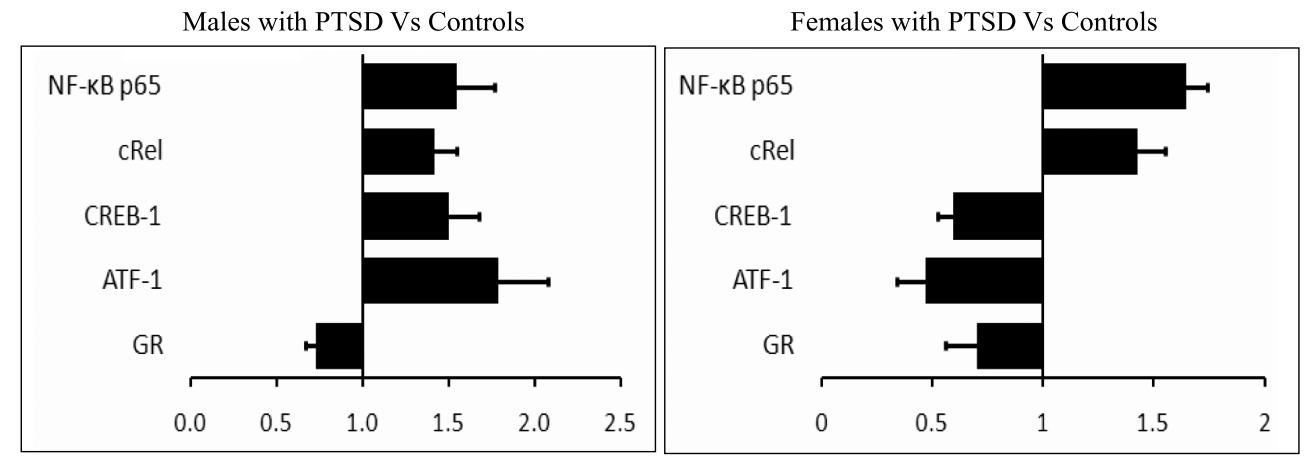

Fig. 2. Fold-difference in the prevalence of response elements for NF- $\kappa$ B/Rel, CREB/ATF and GR in the promoter regions of genes differentially expressed in men and women with PTSD compared with controls. Results indicate significant up-regulation of genes with response elements for $\mathrm{NF}-\kappa \mathrm{B} / \mathrm{Rel}$ and CREB/ATF and significant down-regulation of genes with response elements for GR in men with PTSD compared with age-, sexand trauma-matched controls $(p<0.05$; Fig. 2a). Results also indicate significant up-regulation of genes with response elements for NF- $\kappa$ B/Rel, but significant down-regulation of genes with response elements for CREB/ATF in women with PTSD compared with age- and sex-matched controls $(p<0.05)$. Finally, genes with response elements for GR tended to be down-regulated in women with PTSD, but this effect was not significant $(p=0.11$; Fig. $2 \mathrm{~b})$.

different on marital status (PTSD+ 70\% single, 20\% married and $10 \%$ divorced; Control $75 \%$ single and $25 \%$ married; $p=0.57$ ). Both groups were ethnically heterogeneous, including African American, Asian and White participants in the PTSD+ group and Asian and White participants in the control groups. There were no significant group differences in ethnicity ( $p=$ 0.67). More detailed demographic information can be found in [39]. Trauma exposure and PTSD-related information is presented in Table 1 where it can be seen that only one control participants had been exposed to traumatic events of sufficient severity to fulfill Criterion A1 and A2 for assessment of current PTSD symptoms. No control participants fulfilled diagnostic criteria for current or lifetime PTSD.

\section{Female transcriptional control pathways}

Using a cutoff point of 1.3 fold difference in gene expression levels, 36 up-regulated and 16 down-regulated genes were included in analyses comparing female par- 
ticipants with PTSD with age- and sex-matched controls (Supplementary Table 2 and cf. [39]).

Among women with PTSD, there was significant upregulation of genes with response elements for the NF$\kappa \mathrm{B} /$ Rel family of transcription factors, RelA (1.65-fold difference in V\$NFKAPPAB65_01, SE $=0.10, p<$ $0.001)$ and c-Rel (1.43-fold difference in V\$CREL_01, $\mathrm{SE}=0.13, p=0.008)$. Second, there was significant down-regulation of genes with response elements for CREB-1 (0.60-fold difference in V\$CREB_01, SE $=0.07, p=0.002)$ and ATF-1 (0.47-fold difference in V\$ATF_01, $\mathrm{SE}=0.13, p=0.002)$. Although there were no significant differences in expression of genes bearing response elements for GR, expression of such genes tended to be down-regulated in women with PTSD compared with controls (0.70-fold difference in V\$GR_Q6, SE $=0.14, p=0.11)$. Figure $2 b$ illustrates these findings.

In exploratory analyses, we also found significant up-regulation of genes with response elements for NF- $\kappa$ B1 (2.06-fold difference in V\$NFKAPPAB50_01, $\mathrm{SE}=0.30, p=0.01 ; 1.38$-fold difference in V\$NFKAPPAB_01, SE $=0.18, p=0.04)$. Second, there was significant down-regulation of genes with response elements for additional CREB/ATF transcription factors (0.39-fold difference in V\$CREBP1_Q2; $\mathrm{SE}=0.11, p=0.005)$.

\section{Discussion}

The present study represents the first demonstration of altered transcriptional control of immune cell gene expression in PTSD. Compared with trauma-exposed controls, men with PTSD had significant up-regulation of target genes for NF- $\kappa \mathrm{B}$ and CREB/ATF and significant down-regulation of target genes for GR. These results indicate increased inflammatory and adrenergic signaling in conjunction with decreased glucocorticoid signaling in men with PTSD. Comparing our small sample of women with PTSD with the mixed sample of trauma-exposed and non-trauma-exposed controls, we found significant up-regulation of target genes for NF- $\kappa \mathrm{B}$, but significant down-regulation of target genes for CREB/ATF and non-significant down-regulation of target genes for GR. These results indicate increased inflammatory and decreased adrenergic signaling in women with trauma exposure and PTSD. Differences in CREB/ATF signaling in males versus females could be due to gender effects on these systems or due to differences in characteristics of our control groups. Overall, our results indicate that PTSD is associated with altered transcriptional control of monocyte gene expression, specifically with increased inflammatory signaling that could plausibly contribute to increased physical disease risk in PTSD.

Recent demonstrations of elevated inflammatory activity in PTSD have highlighted inflammatory activity as a potential mechanism accounting for increased physical disease risk $[22,53,57]$. However, not all studies have reported elevated inflammatory activity in individuals with PTSD [24]. Further, some of the largest studies that have demonstrated this association have included control participants who were not trauma exposed and the samples have included pooled groups of men and women with PTSD [22,53]. Primary analysis of the specific genes differentially expressed in the present sample was not suggestive of elevated inflammatory activity in men with PTSD [39]. However, the present analysis indicates that both men and women with PTSD demonstrate up-regulation of target genes for the transcription factors RelA and c-Rel from the $\mathrm{NF}-\kappa \mathrm{B}$ family, indicating elevated pro-inflammatory signaling to monocytes in these samples with PTSD. Given matching for trauma exposure in the male sample, the data also provide preliminary evidence that PTSD is associated with increased inflammatory signaling over and above trauma exposure, at least in men. Inflammatory activity has been causally implicated in the development of chronic diseases including cardiovascular disease, autoimmune disorders and cancer, as well as neurodegenerative diseases and some forms of major depressive disorder $[9,14,32,36,47]$. Thus, elevated inflammatory activity in PTSD could increase risk for both physical and mental disorders.

The finding that target genes for CREB/ATF were up-regulated in men with PTSD compared with traumaexposed controls is in line with a large body of research indicating increased SNS activation in PTSD [40,65]. Our data showing increased CREB/ATF signaling in men with PTSD is consistent with prior observations of increased SNS activity and elevated levels of circulating catecholamines [56]. However, we found that target genes for CREB/ATF were significantly downregulated in women with trauma exposure and PTSD, indicating decreased CREB/ATF signaling in monocytes in the female sample. Sex hormone differences may influence stress effects on CREB and thereby influence the expression of target genes for CREB/ATF [28, $29,55]$. However, it is also possible that differences in trauma exposure between the male and female control groups contributed to the findings. 
Regardless of why differences in expression of target genes for CREB/ATF occurred in the male and female samples, the difference in CREB/ATF signaling between the two samples may have important implications for the regulation of inflammatory activity in PTSD. Previous research indicates that a mixed sample of men and women with trauma exposure and PTSD demonstrated elevation of pro-inflammatory cytokines as well as elevation of levels of the anti-inflammatory cytokine interleukin-10 (IL-10), but it was not clear if both male and female participants showed this effect [22]. Binding of CREB/ATF in monocytes may contribute to this up-regulation of IL-10 production [ 45 , 46]. Because IL-10 has immunosuppressive and antiinflammatory properties [35], this process could attenuate stress-related inflammatory activity and reduce risk for inflammatory disease. Thus, increased CREB/ATF signaling may contribute to regulating PTSD-related increases in inflammatory activity.

Our analysis indicated significant down-regulation of target genes for GR in men with PTSD and nonsignificant down-regulation of target genes for GR in women with trauma exposure and PTSD. As an index of lower glucocorticoid signaling to the monocyte transcriptome in PTSD, this finding is in line with a substantial body of research showing lower circulating levels of cortisol in PTSD [37,60,63,67]. However, an almost equally large body of previous research supported the hypothesis of enhanced negative feedback or enhanced GR sensitivity in PTSD [42,61,66,68]. While it is difficult to draw firm conclusions about such GR sensitivity without knowing the level of circulating cortisol in our participants, our data are not supportive of greater GR sensitivity on monocytes in PTSD.

The present paper highlights the utility of analyzing gene expression data at multiple levels. In our previous analysis of the present data, we were interested in the expression of individual genes and gene ontologies and therefore applied a stringent threshold, examining only genes differentially expressed $\geqslant 50 \%$ between groups. The results showed a general decrease in expression of all genes in monocytes in the male sample, and no evidence of increased inflammation [39]. In the present study, we were less interested in individual gene probes and more interested in upstream patterns of transcriptional control by a limited number of $a$ priori selected specific transcription factors involved in inflammatory, adrenergic and glucocorticoid signaling. Thus, in the present research, we used a more permissive cutoff and included genes differentially expressed $\geqslant 30 \%$ between groups. While the earlier analysis indicated a gener- al pattern of down-regulation of genes in monocytes, the present analysis indicates a propensity towards proinflammatory signaling in both men and women with PTSD. Further research will be necessary to shed light on how such patterns of gene expression and signaling are manifest at the level of circulating proteins. Taken together, these data underscore the importance of decisions regarding stringency levels in high-throughput data analytic techniques, and additionally highlight the value of employing analysis at different levels in research examining the influence of psychosocial factors on biological mediators of disease [11].

The major limitations of our study relate to our female sample and include the small sample size as well as the mixed sample of trauma exposed and unexposed controls. We consider data on this female sample preliminary and in need of replication. However, findings in relation to our male sample must also be interpreted in the context of some limitations. First, while males with PTSD were matched with controls for trauma exposure, it is difficult to conclude with certainty that the men with PTSD and controls experienced trauma equivalent in severity or duration. Second, the present analysis would benefit from the availability of data on circulating levels of the various proteins of interest to our analysis - specifically levels of pro- and anti-inflammatory cytokines, catecholamines and cortisol - in both male and female samples. Third, while we believe that the focus on a homogenous population of immune cells is a major strength of our research, the present findings may not apply to other immune cell populations. Overall, the results suggest a research agenda to address questions unanswered by this study. First, replication of the present research in a larger sample of women with PTSD and age-, sex- and trauma-matched controls would help to shed light on the relative impact of sex and trauma exposure on the divergent CREB/ATF signaling patterns observed between male and female samples in our study. Second, the relationship between severity of PTSD and signaling to immune cells could be addressed by conducting prospective studies that involve repeated assessments of PTSD symptom severity or non-pharmacological treatment of PTSD to avoid medication confounds. Another potentially worthwhile avenue of research is to examine in vitro adrenergic and glucocorticoid regulation of gene expression in monocytes from individuals with and without PTSD.

To our knowledge, the present analysis provides the first ever demonstration of altered transcriptional control of monocyte gene expression in PTSD. Our da- 
ta suggest that men with PTSD have increased NF$\kappa \mathrm{B}$ signaling, increased $\mathrm{CREB} / \mathrm{ATF}$ signaling and reduced GR signaling to the monocyte transcriptome. Our data additionally suggest that women with trauma exposure and PTSD have increased NF- $\kappa \mathrm{B}$ signaling and decreased CREB/ATF signaling to the monocyte transcriptome. These findings contribute to a growing body of literature showing altered transcriptional control pathways in association with chronic psychological stress and psychiatric symptoms $[12,30,34]$. The findings also shed light on how the complex patterns of biological dysregulation in PTSD are manifest at the level of the monocyte. In sum, the data provide evidence that altered transcription factor signaling mechanisms in monocytes could contribute to elevated inflammatory activity in PTSD.

\section{Acknowledgements}

We thank Cyrus Calosing for excellent technical assistance and Joshua D. Woolley, MD/PhD for comments on an earlier draft of this manuscript. This research was supported in part by grants from the Department of Defense (W81XWH-05-2-0094), the Mental Illness Research and Education Clinical Center (MIRECC) of the US Veterans Health Administration, and the National Institute for Mental Health (TCN: R01 MH73978). This material is the result of work supported with resources and the use of facilities at the Veterans Administration Medical Center, San Francisco California.

\section{Supplementary material}

Supplementary data can be found on: www.aoifeodonovan.net

\section{References}

[1] S. Basak and A. Hoffmann, Crosstalk via the NF-kappaB signaling system. Cytokine and Growth Factor Reviews 19 (2008), 187-197.

[2] A. Bierhaus, J. Wolf, M. Andrassy, N. Rohleder, P.M. Humpert, D. Petrov, R. Ferstl, M. von Eynatten, T. Wendt, G. Rudofsky, M. Joswig, M. Morcos, M. Schwaninger, B. McEwen, C. Kirschbaum and P.P. Nawroth, A mechanism converting psychosocial stress into mononuclear cell activation, Proceedings of the National Academy of Sciences of the U S A 100 (2003), 1920-1925.
[3] D.D. Blake, F.W. Weathers, L.M. Nagy, D.G. Kaloupek, F.D. Gusman, D.S. Charney and T.M. Keane, The development of a clinician-administered PTSD scale, Journal of Traumatic Stress 8 (1995), 75-90.

[4] J.A. Boscarino, Posttraumatic stress disorder and physical illness: results from clinical and epidemiologic studies, Annals of the New York Academy of Sciences 1032 (2004), 141-153.

[5] J.A. Boscarino, A prospective study of PTSD and early-age heart disease mortality among Vietnam veterans: implications for surveillance and prevention, Psychosomatic Medicine 70 (2008), 668-676.

[6] J.A. Boscarino, Psychobiologic predictors of disease mortality after psychological trauma: implications for research and clinical surveillance, The Journal of Nervous and Mental Disease 196 (2008), 100-107.

[7] J.A. Boscarino and C.R. Figley, The impact of repression, hostility, and post-traumatic stress disorder on all-cause mortality: a prospective 16-year follow-up study, The Journal of Nervous and Mental Disease 197 (2009), 461-466.

[8] J.A. Boscarino, C.W. Forsberg and J. Goldberg, A twin study of the association between PTSD symptoms and rheumatoid arthritis, Psychosomatic Medicine 72 (2010), 481-486.

[9] M. Cesari, B.W. Penninx, A.B. Newman, S.B. Kritchevsky, B.J. Nicklas, K. Sutton-Tyrrell, S.M. Rubin, J. Ding, E.M. Simonsick, T.B. Harris and M. Pahor, Inflammatory markers and onset of cardiovascular events: results from the Health ABC study, Circulation 108 (2003), 2317-2322.

[10] B.E. Cohen, C.R. Marmar, T.C. Neylan, N.B. Schiller, S. Ali and M.A. Whooley, Posttraumatic stress disorder and healthrelated quality of life in patients with coronary heart disease: findings from the Heart and Soul Study, Archives of General Psychiatry 66 (2009), 1214-1220.

[11] S.W. Cole, J.M. Arevalo, R. Takahashi, E.K. Sloan, S.K. Lutgendorf, A.K. Sood, J.F. Sheridan and T.E. Seeman, Computational identification of gene-social environment interaction at the human IL6 locus, Proceedings of the National Academy of Sciences of the U S A 107 (2010), 5681-5686.

[12] S.W. Cole, L.C. Hawkley, J.M. Arevalo, C.Y. Sung, R.M. Rose and J.T. Cacioppo, Social regulation of gene expression in human leukocytes, Genome Biology 8 (2007), R189.

[13] S.W. Cole, W. Yan, Z. Galic, J. Arevalo and J.A. Zack, Expression-based monitoring of transcription factor activity: the TELiS database, Bioinformatics 21 (2005), 803-810.

[14] R. Dantzer, J.C. O'Connor, G.G. Freund, R.W. Johnson and K.W. Kelley, From inflammation to sickness and depression: when the immune system subjugates the brain, Nature Reviews Neuroscience 9 (2008), 46-56.

[15] I.J. Elenkov and G.P. Chrousos, Stress hormones, Th1/Th2 patterns, pro/anti-inflammatory cytokines and susceptibility to disease, Trends in Endocrinology and Metabolism 10 (1999), 359-368.

[16] I.J. Elenkov, D.A. Papanicolaou, R.L. Wilder and G.P. Chrousos, Modulatory effects of glucocorticoids and catecholamines on human interleukin-12 and interleukin-10 production: clinical implications, Proceedings of the Association of American Physicians 108 (2000), 374-381.

[17] M.B. First, R.L. Spitzer, J.B.W. Williams and M. Gibbon, Structured Clinical Interview for DSM-IV (SCID-I; Patient Version ed.). 1996, New York: New York State Psychiatric Institute, Biometrics Research.

[18] R.C. Gentleman, V.J. Carey, D.M. Bates, B. Bolstad, M. Dettling, S. Dudoit, B. Ellis, L. Gautier, Y. Ge, J. Gentry, K. Hornik, T. Hothorn, W. Huber, S. Iacus, R. Irizarry, F. Leisch, C. Li, M. Maechler, A.J. Rossini, G. Sawitzki, C. Smith, G. 
Smyth, L. Tierney, J.Y. Yang and J. Zhang, Bioconductor: open software development for computational biology and bioinformatics, Genome Biology 5 (2004), R80.

[19] Y. Gidron, T. Armon, H. Gilutz and M. Huleihel, Psychological factors correlate meaningfully with percent-monocytes among acute coronary syndrome patients, Brain, Behavior, and Immunity 17 (2003), 310-315.

[20] L.A. Grisanti, J. Evanson, E. Marchus, H. Jorissen, A.P. Woster, W. DeKrey, E.R. Sauter, C.K. Combs and J.E. Porter, Pro-inflammatory responses in human monocytes are beta1adrenergic receptor subtype dependent, Molecular Immunology 47 (2010), 1244-1254.

[21] M.S. Hayden and S. Ghosh, Shared principles in NF-kappaB signaling, Cell 132 (2008), 344-362.

[22] E.A. Hoge, K. Brandstetter, S. Moshier, M.H. Pollack, K.K. Wong and N.M. Simon, Broad spectrum of cytokine abnormalities in panic disorder and posttraumatic stress disorder, Depression and Anxiety 26 (2009), 447-455.

[23] S.S. Inslicht, C.R. Marmar, T.C. Neylan, T.J. Metzler, S.L. Hart, C. Otte, S.E. McCaslin, G.L. Larkin, K.B. Hyman and A. Baum, Increased cortisol in women with intimate partner violence-related posttraumatic stress disorder, Psychoneuroendocrinology 31 (2006), 825-838.

[24] N. Kawamura, Y. Kim and N. Asukai, Suppression of cellular immunity in men with a past history of posttraumatic stress disorder, American Journal of Psychiatry 158 (2001), 484486.

[25] M. Kellner, K. Wiedemann, A. Yassouridis, R. Levengood, L.S. Guo, F. Holsboer and R. Yehuda, Behavioral and endocrine response to cholecystokinin tetrapeptide in patients with posttraumatic stress disorder, Biological Psychiatry 47 (2000), 107-111.

[26] R. Knuppel, P. Dietze, W. Lehnberg, K. Frech and E. Wingender, TRANSFAC retrieval program: a network model database of eukaryotic transcription regulating sequences and proteins, J Comput Biol 1 (1994), 191-198.

[27] M. Lekander, J. Axen, U. Knutsson, C. Olgart Hoglund, S. Werner, A.C. Wikstrom and P. Stierna, Cytokine inhibition after glucocorticoid exposure in healthy men with low versus high basal cortisol levels, Neuroimmunomodulation 16 (2009), 245-250.

[28] Y. Lin, G.J. Ter Horst, R. Wichmann, P. Bakker, A. Liu, X. Li and $C$. Westenbroek, Sex differences in the effects of acute and chronic stress and recovery after long-term stress on stressrelated brain regions of rats, Cerebral Cortex 19 (2009), 19781989.

[29] Y. Lin, C. Westenbroek, P. Bakker, J. Termeer, A. Liu, X. Li and G.J. Ter Horst, Effects of long-term stress and recovery on the prefrontal cortex and dentate gyrus in male and female rats, Cerebral Cortex 18 (2008), 2762-2774.

[30] S.K. Lutgendorf, K. DeGeest, C.Y. Sung, J.M. Arevalo, F. Penedo, J. Lucci, 3rd, M. Goodheart, D. Lubaroff, D.M. Farley, A.K. Sood and S.W. Cole, Depression, social support, and beta-adrenergic transcription control in human ovarian cancer, Brain, Behavior and Immunity 23 (2009), 176-183.

[31] M. Maes, D.R. Van Bockstaele, A. Gastel, C. Song, C. Schotte, H. Neels, I. DeMeester, S. Scharpe and A. Janca, The effects of psychological stress on leukocyte subset distribution in humans: evidence of immune activation, Neuropsychobiology 39 (1999), 1-9.

[32] P.L. McGeer and E.G. McGeer, Inflammation and the degenerative diseases of aging, Annals of the New York Academy of Sciences 1035 (2004), 104-116.
[33] F. Middle, I. Jones, E. Robertson, C. Lendon and N. Craddock, Tumour necrosis factor alpha and bipolar affective puerperal psychosis, Psychiatric Genetics 10 (2000), 195-198.

[34] G.E. Miller, E. Chen, J. Sze, T. Marin, J.M.G. Arevalo, R. Doll, R. Ma and S.W. Cole, A functional genomic fingerprint of chronic stress in humans: Blunted glucocorticoid and increased NF-kB signaling, Biological Psychiatry (2008).

[35] K.W. Moore, R. de Waal Malefyt, R.L. Coffman and A. O'Garra, Interleukin-10 and the interleukin-10 receptor, Annual Review of Immunology 19 (2001), 683-765.

[36] S.F. Moss and M.J. Blaser, Mechanisms of disease: Inflammation and the origins of cancer, Nature Clinical Practice Oncology 2 (2005), 90-97.

[37] T.C. Neylan, A. Brunet, N. Pole, S.R. Best, T.J. Metzler, R. Yehuda and C.R. Marmar, PTSD symptoms predict waking salivary cortisol levels in police officers, Psychoneuroendocrinology 30 (2005), 373-381.

[38] T.C. Neylan, C. Otte, R. Yehuda and C.R. Marmar, Neuroendocrine regulation of sleep disturbances in PTSD, Ann NY Acad Sci 1071 (2006), 203-215.

[39] T.C. Neylan, B. Sun, H. Rempel, J. Ross, M. Lenoci, A. O'Donovan and L. Pulliam, Suppressed peripheral monocyte gene profile in healthy men with PTSD. Under submission (2010).

[40] T. O'Donnell, K.M. Hegadoren and N.C. Coupland, Noradrenergic mechanisms in the pathophysiology of post-traumatic stress disorder, Neuropsychobiology 50 (2004), 273-283.

[41] L.A. O'Neill and C. Kaltschmidt, NF-kappa B: a crucial transcription factor for glial and neuronal cell function, Trends in Neurosciences 20 (1997), 252-258.

[42] C. Otte, M. Lenoci, T. Metzler, R. Yehuda, C.R. Marmar and T.C. Neylan, Hypothalamic-pituitary-adrenal axis activity and sleep in posttraumatic stress disorder, Neuropsychopharmacology 30 (2005), 1173-1180.

[43] C. Otte, M. Lenoci, T. Metzler, R. Yehuda, C.R. Marmar and T.C. Neylan, Effects of metyrapone on hypothalamicpituitary-adrenal axis and sleep in women with post-traumatic stress disorder. Biological Psychiatry 61 (2007), 952-956.

[44] R.K. Pitman and S.P. Orr, Twenty-four hour urinary cortisol and catecholamine excretion in combat-related posttraumatic stress disorder, Biological Psychiatry 27 (1990), 245-247.

[45] C. Platzer, W. Docke, H. Volk and S. Prosch, Catecholamines trigger IL-10 release in acute systemic stress reaction by direct stimulation of its promoter/enhancer activity in monocytic cells, Journal of Neuroimmunology 105 (2000), 31-38.

[46] C. Platzer, E. Fritsch, T. Elsner, M.H. Lehmann, H.D. Volk and S. Prosch, Cyclic adenosine monophosphate-responsive elements are involved in the transcriptional activation of the human IL-10 gene in monocytic cells, European Journal of Immunology 29 (1999), 3098-3104.

[47] A.D. Pradhan, J.E. Manson, N. Rifai, J.E. Buring and P.M. Ridker, C-reactive protein, interleukin 6, and risk of developing type-2 diabetes mellitus, Journal of the American Medical Association 286 (2001), 327-334.

[48] L. Pulliam, B. Sun and H. Rempel, Invasive chronic inflammatory monocyte phenotype in subjects with high HIV-1 viral load. Journal of Neuroimmunology 157 (2004), 93-98.

[49] S.U. Qureshi, J.M. Pyne, K.M. Magruder, P.E. Schulz and M.E. Kunik, The link between post-traumatic stress disorder and physical comorbidities: a systematic review, The Psychiatric Quarterly 80 (2009), 87-97.

[50] C.L. Raison and A.H. Miller, When not enough is too much: the role of insufficient glucocorticoid signaling in the patho- 
physiology of stress-related disorders, Am J Psychiatry 160 (2003), 1554-1565.

[51] P. Rontgen, A. Sablotzki, A. Simm, R.E. Silber and E. Czeslick, Effect of catecholamines on intracellular cytokine synthesis in human monocytes, Eur Cytokine Netw 15 (2004), 14-23.

[52] C. Spitzer, S. Barnow, H. Volzke, U. John, H.J. Freyberger and H.J. Grabe, Trauma, posttraumatic stress disorder, and physical illness: findings from the general population, Psychosom Med 71 (2009), 1012-1017.

[53] C. Spitzer, S. Barnow, H. Volzke, H. Wallaschofski, U. John, H.J. Freyberger, B. Lowe and H.J. Grabe, Association of posttraumatic stress disorder with low-grade elevation of Creactive protein: evidence from the general population, Journal of Psychiatric Research 44 (2010), 15-21.

[54] R.D.C. Team, R: A language and environment for statistical computing, 2009 cited; Available from: http://www.Rproject.org.

[55] G.J. Ter Horst, R. Wichmann, M. Gerrits, C. Westenbroek and Y. Lin, Sex differences in stress responses: focus on ovarian hormones, Physiol Behav 97 (2009), 239-249.

[56] E.J. Videlock, T. Peleg, R. Segman, R. Yehuda, R.K. Pitman and A.Y. Shalev, Stress hormones and post-traumatic stress disorder in civilian trauma victims: a longitudinal study. Part II: the adrenergic response, International Journal of Neuropsychopharmacology 11 (2008), 373-380.

[57] R. von Kanel, U. Hepp, B. Kraemer, R. Traber, M. Keel, L. Mica and U. Schnyder, Evidence for low-grade systemic proinflammatory activity in patients with posttraumatic stress disorder, Journal of Psychiatric Research 41 (2007), 744-752.

[58] C. Wietek and L.A. O'Neill, Diversity and regulation in the NF-kappaB system, Trends in Biochemical Sciences 32 (2007), 311-319.

[59] K. Yaffe, E. Vittinghoff, K. Lindquist, D. Barnes, K.E. Covinsky, T. Neylan, M. Kluse and C. Marmar, Posttraumatic stress disorder and risk of dementia among US veterans, Archives of
General Psychiatry 67 (2010), 608-613.

[60] R. Yehuda, Status of glucocorticoid alterations in posttraumatic stress disorder, Annals of the New York Academy of Sciences 1179 (2009), 56-69.

[61] R. Yehuda, G. Cai, J.A. Golier, C. Sarapas, S. Galea, M. Ising, T. Rein, J. Schmeidler, B. Muller-Myhsok, F. Holsboer and J.D. Buxbaum, Gene expression patterns associated with posttraumatic stress disorder following exposure to the World Trade Center attacks, Biological Psychiatry 66 (2009), 708711.

[62] R. Yehuda, J.A. Golier, S.L. Halligan, M. Meaney and L.M. Bierer, The ACTH response to dexamethasone in PTSD, American Journal of Psychiatry 161 (2004), 1397-1403.

[63] R. Yehuda, J.A. Golier and S. Kaufman, Circadian rhythm of salivary cortisol in Holocaust survivors with and without PTSD, American Journal of Psychiatry 162 (2005), 998-1000.

[64] R. Yehuda, B. Kahana, K. Binder-Brynes, S.M. Southwick, J.W. Mason, and E.L. Giller, Low urinary cortisol excretion in Holocaust survivors with posttraumatic stress disorder, American Journal of Psychiatry 152 (1995), 982-986.

[65] R. Yehuda and J. LeDoux, Response variation following trauma: a translational neuroscience approach to understanding PTSD, Neuron 56 (2007), 19-32.

[66] R. Yehuda, S.M. Southwick, J.H. Krystal, D. Bremner, D.S. Charney and J.W. Mason, Enhanced suppression of cortisol following dexamethasone administration in posttraumatic stress disorder, American Journal of Psychiatry 150 (1993), 83-86.

[67] R. Yehuda, S.M. Southwick, G. Nussbaum, V. Wahby, E.L. Giller, Jr. and J.W. Mason, Low urinary cortisol excretion in patients with posttraumatic stress disorder, Journal of Nervous and Mental Disease 178 (1990), 366-369.

[68] R. Yehuda, R.K. Yang, M.S. Buchsbaum and J.A. Golier, Alterations in cortisol negative feedback inhibition as examined using the ACTH response to cortisol administration in PTSD, Psychoneuroendocrinology 31 (2006), 447-451. 


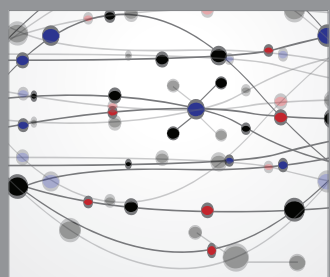

The Scientific World Journal
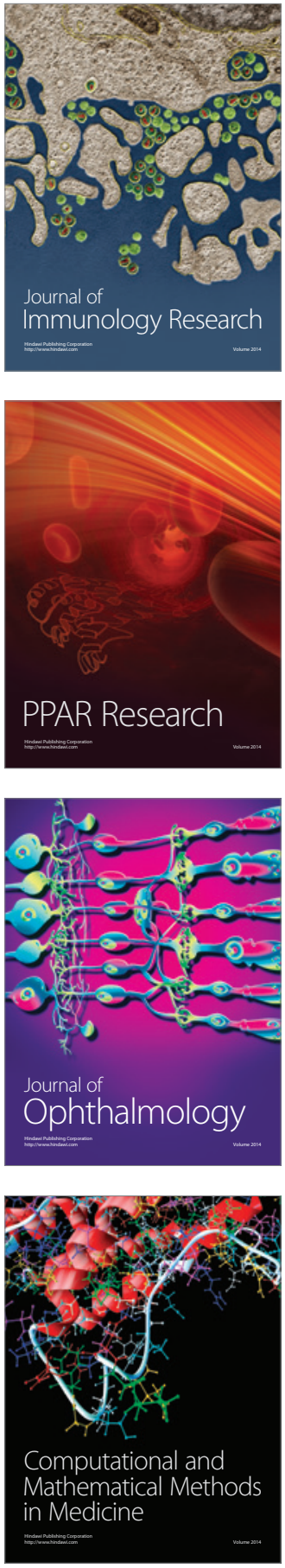

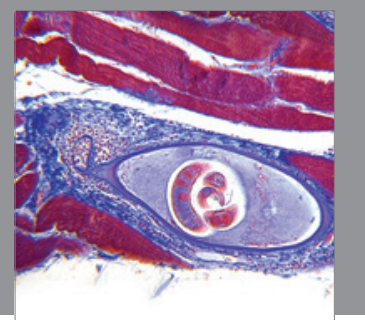

Gastroenterology

Research and Practice
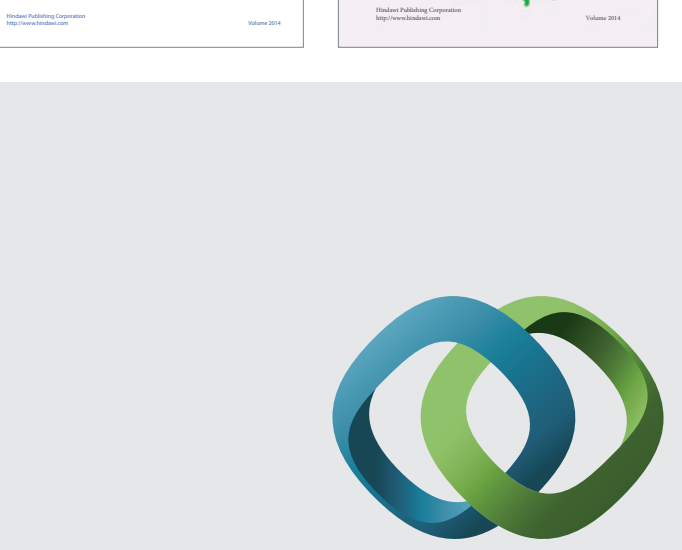

\section{Hindawi}

Submit your manuscripts at

http://www.hindawi.com
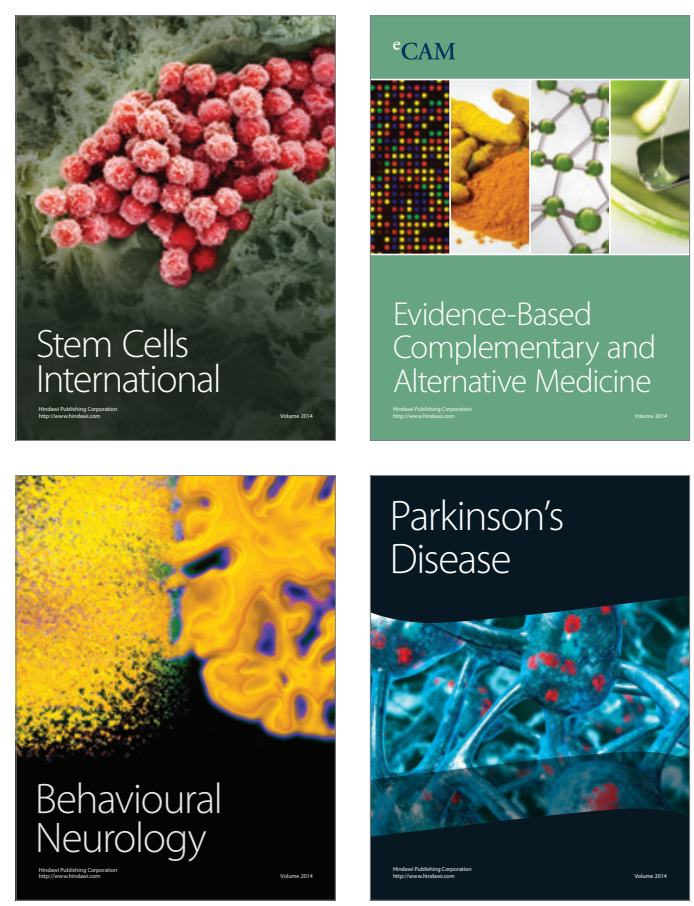

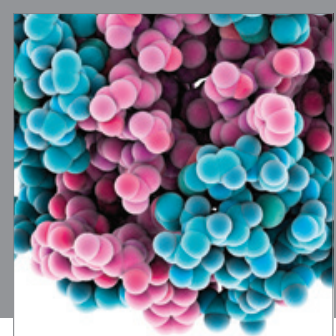

Journal of
Diabetes Research

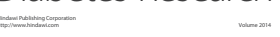

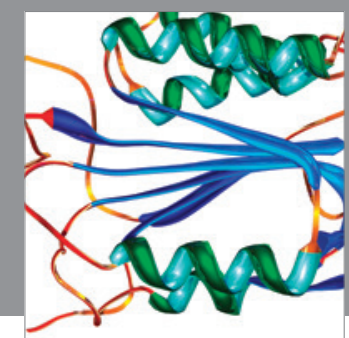

Disease Markers
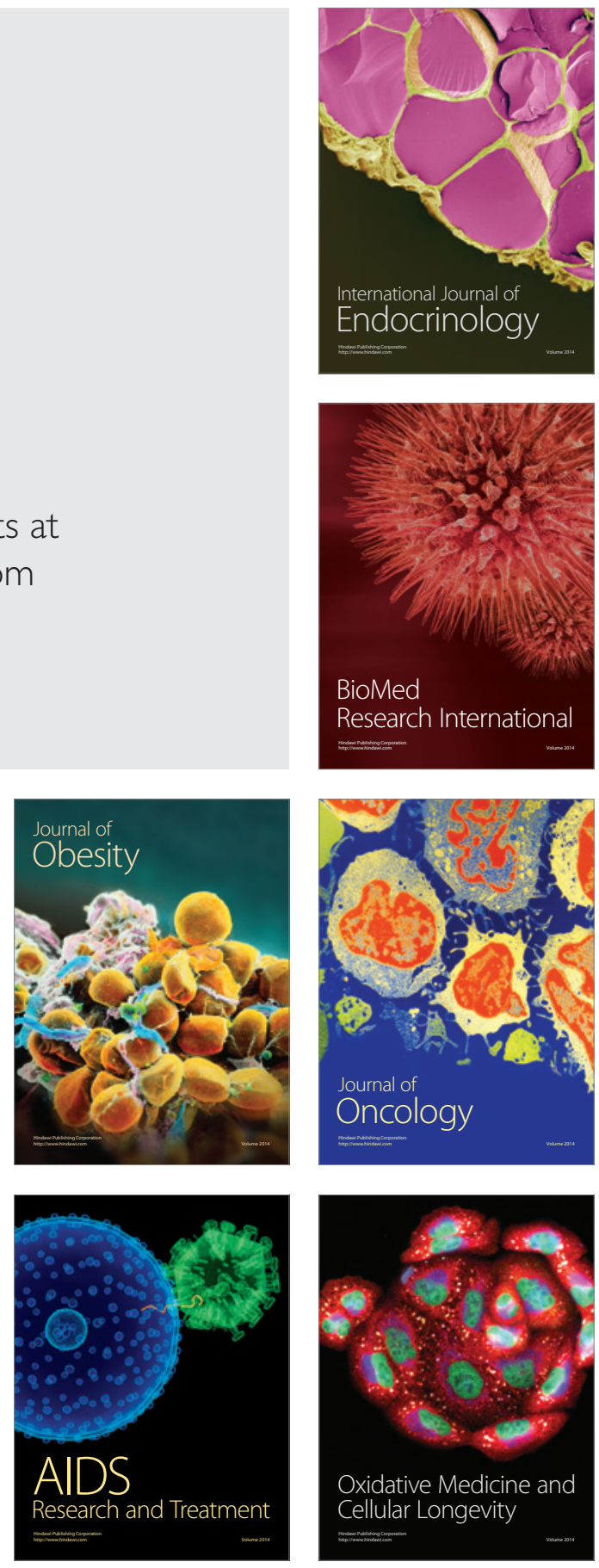